EPJ Web of Conferences 101, 01001 (2015)

DOI: 10.1051/epjconf/ 201510101001

C) Owned by the authors, published by EDP Sciences, 2015

\title{
Stellar evolution as seen by mixed modes
}

\author{
Benoît Mosser ${ }^{1, \mathrm{a}}$ \\ LESIA, CNRS, Université Pierre et Marie Curie, Université Denis Diderot, Observatoire de Paris, \\ 92195 Meudon cedex, France
}

\begin{abstract}
The detection of mixed modes in subgiants and red giants allows us to monitor stellar evolution from the main sequence to the asymptotic giant branch and draw seismic evolutionary tracks. Quantified asteroseismic definitions that characterize the change in the evolutionary stages have been defined. This seismic information can now be used for stellar modelling, especially for studying the energy transport in the helium burning core or for specifying the inner properties of stars all along their evolution. Modelling will also allow us to study stars identified in the helium subflash stage, high-mass stars either arriving or quitting the secondary clump, or stars that could be in the blue-loop stage.
\end{abstract}

\section{Mixed modes}

Oscillation mixed modes have shown their capability to probe the interior structure of subgiants and red giants $[3,16]$. As mixed modes result from the coupling of gravity and pressure wave, their oscillation pattern is based on both the large frequency separation $\Delta v$ and the gravity period spacing $\Delta \Pi_{1}$. The identification of the mixed mode pattern then provides information on the stellar envelope and on the core. This seismic information is so precise that precise evolutionary tracks can be derived [19]. Here we show how such seismic information can be obtained and how it translates in an HR diagram.

The determinations of $\Delta v$ and $\Delta \Pi_{1}$ are based on asymptotic expansions $[14,16]$. For pure pressure modes, the expansion is valid when the oscillation radial order is large enough. However, observable modes excited around the frequency $v_{\max }$ of the maximum oscillation signal have significantly lower frequencies than the asymptotic regime. By chance, the equivalent radial order $n_{\max }$, approximately corresponding to $v_{\max } / \Delta v$, is large enough to insure that the oscillation pattern is close to the secondorder asymptotic pattern, so that deriving the asymptotic parameters is possible $[14,18]$. The use of this method allows us to cancel the modulation of the radial oscillation pattern due to glitches (Vrard et al., these proceedings). The mean accuracy of the large separation is about $0.04 \mu \mathrm{Hz}$ (this translates into a $1 \%$-relative precision at the red clump).

Mixed modes are fitted with the asymptotic expansion based on the period spacing $\Delta \Pi_{1}[22,16]$. The precise fit must account for any rotational splitting [1,17,9,7]. For red giants, the observed gravity mode orders are high enough to ensure the validity of the asymptotic expansion, hence a high precision of the asymptotic global parameters as soon as mixed modes are clearly visible [15]. The period spacing $\Delta \Pi_{1}$ is then determined with a precision better than $2.5 \%$ [16] (and in many cases better than $0.5 \%$ ). For subgiants, the gravity orders of the few observed mixed modes, are small, down to 2 in many cases. This complicates the fit and requires fitting all parameters of the asymptotic expansion: the period spacing and the coupling (also free parameters for red giants), but also the phase offset (assumed to be 0 for red giants). The accuracy of the asymptotic expansion had therefore to be tested: comparison with a different approach $[3,4]$ indicates agreement to within $10 \%$. As a result, we could fit the mixed modes in the subgiants observed with Kepler [6], except in very faint stars with low signal-to-noise ratios. We are confident that the asymptotic expansion is qualitatively correct, although

a e-mail: benoit.mosser@obspm.fr

This is an Open Access article distributed under the terms of the Creative Commons Attribution License 4.0, which permits unrestricted use, distribution, and reproduction in any medium, provided the original work is properly cited. 

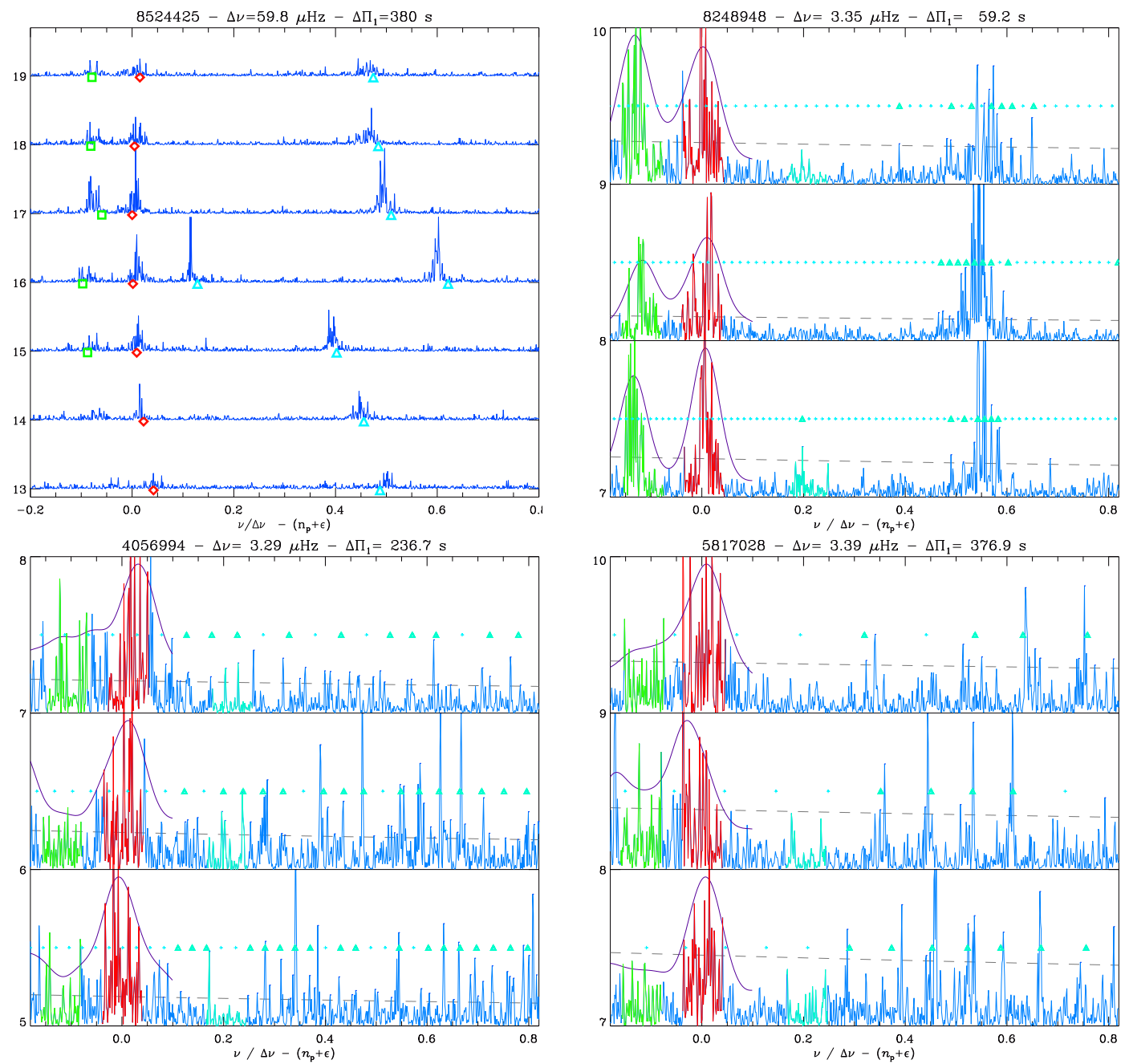

Fig. 1. Example of a fit to the mixed modes. On the x-axis, the frequency is represented in $\Delta v$ units, corrected for the pressure radial order $n_{\mathrm{p}}$ and the offset $\varepsilon$, so that radial modes (in red) are located near the abscissa value 0 . The pressure oscillation pattern and the large frequency separation $\Delta v$ are derived from the radial modes (red diamonds); the period spacing $\Delta \Pi_{1}$ is derived from the fit of the dipole mixed mode pattern. a: Subgiant oscillation spectrum with dipole modes indicated by blue triangles and quadrupole modes by green squares. b: Échelle spectrum of an RGB star with a low $\Delta \Pi_{1}$. The purple line superimposed on the quadrupole and radial modes correspond to a smoothed envelope of the spectrum. The grey dashed line shows the background level multiplied by a factor of 8 . The plus symbols indicate the position of the asymptotic mixed modes and are highlighted with a triangle symbol when they correspond to reliably detected peaks. On the red giant branch (RGB), the number of mixed modes with significant height is small, which makes the identification of $\Delta \Pi_{1}$ challenging c: Star starting to climb the asymptotic giant branch, with a similar $\Delta v$ but a larger $\Delta \Pi_{1}$ than in case b. d: Star just after the helium flash.

for early subgiants we cannot exclude a small bias between the observed and the asymptotic period spacings $\Delta \Pi_{1}$, similar to that observed for the large frequency separation $\Delta v$ [18]. Currently, theoretical models reproduce the period spacings on the red giant branch but not in the red clump [11,12,21], unless extra mixing is considered [20].

Figure 1 shows the fit of the mixed mode pattern in a subgiant (Fig. 1a) and in three red giants with similar mean density, hence similar $\Delta v$, but different evolutionary stages, hence different $\Delta \Pi_{1}$. For a a star on the red giant branch (Fig. 1b), mixed modes are only present in a narrow frequency range 

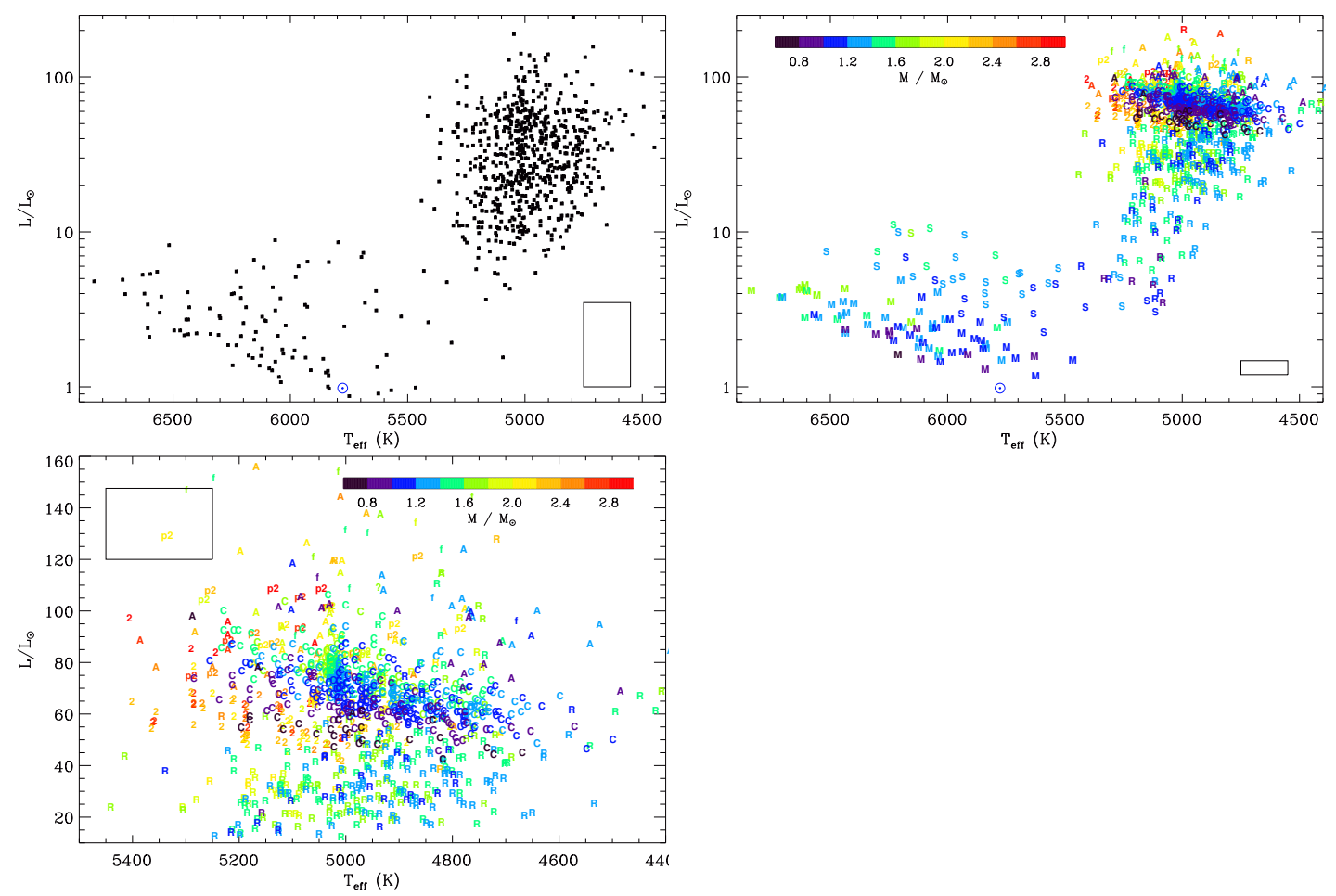

Fig. 2. HR diagrams of stars showing solar-like oscillations observed with Kepler. a, Diagram without any seismic information. The error box in the lower-right corner indicates the typical uncertainty on effective temperature and luminosity for field stars without seismic constraints [10]. b, Same diagram taking the seismic information into account. Evolutionary stages are defined in the text. Mass and luminosity estimates are provided from seismic scaling relations. c, Zoom in the red clump region.

surrounding the frequency of pure pressure modes. As a result, measuring the period spacing is most often impossible, except when the star is seen pole on, so that the rotational multiplets are reduced to the $m=0$ component. On the contrary, a large number of gravity-dominated mixed modes are present in a star starting the ascension of the asymptotic giant branch (Fig. 1c). Stars undergoing the helium flash have a gravity period spacing $\Delta \Pi_{1}$ which is discrepant in the $\Delta \Pi_{1}-\Delta v$ diagram (Fig. 1d).

\section{Evolutionary stages}

Previous work was able to distinguish RGB stars burning hydrogen in a shell from clump stars burning helium in the core $[2,13,21]$. We can now follow stellar evolution from the main-sequence to the asymptotic giant branch (AGB) and provide valuable information for stellar modeling since the $\Delta \Pi_{1}$ $-\Delta v$ diagram exhibits clear mass-dependent evolutionary tracks (Fig. 2 of [19]). Assuming that the density of stars in the $\Delta \Pi_{1}-\Delta v$ diagram is related to the evolution rate, the transition from the subgiant to the RGB stage have been defined by $(\Delta v / 36.5 \mu \mathrm{Hz})\left(\Delta \Pi_{1} / 126 \mathrm{~s}\right)<1$, as the transition from the clump to the early AGB, with $(\Delta v / 3.3 \mu \mathrm{Hz})^{1.5}\left(\Delta \Pi_{1} / 245 \mathrm{~s}\right)<1$.

The different evolutionary stages that could be seismically inferred are shown in a classical HR diagram. Figure 2a, without any seismic information, serves as a reference, whereas Fig. $2 \mathrm{~b}$ takes the seismic information into account. Main-sequence stars (M) have no mixed modes, contrary to subgiants $(\mathbf{S})$ that have a well-developed radiative core. Benefitting from 4-year long time series, RGB stars (R) can be identified at high luminosity. Clump stars (C) show a clear gradient in the HR diagram, with low-mass stars significantly less luminous than more massive stars. Compared to clump stars, secondary clump stars (2) more massive than $1.9 M_{\odot}$ show lower luminosity [8], whereas stars leaving 
the red or secondary clumps and moving towards the AGB (A) have significantly brighter luminosities. The $\Delta \Pi_{1}-\Delta v$ diagram emphasizes also the $\mathbf{p} 2$ stage, corresponding to progenitors of secondary clump stars. These progenitors are significantly more luminous than stars lying in the secondary clump. Apart from these stages, a few outliers are identified as stars starting the helium burning stage in the unstable contraction phase following the helium flash [5] (f).

All transitions between the various stages of evolution, such as hydrogen-core burning, heliumcore burning, or the decrease of helium burning in the core, are marked by changes in the relationship between the frequency and period spacings. For low-mass stars arriving on the RGB, the period spacing varies with the frequency spacing in a close relationship, because the core and envelope structures are closely linked in that stage. The period spacing varies also univocally with the frequency spacing with a mass-independent relationship when helium is exhausted in the degenerate core. On the contrary, a source of energy in the red-clump star core lifts the degeneracy, so that the relation between $\Delta \Pi_{1}$ and $\Delta v$ depends on the mass.

Comparison with modeling will help making the link between these phenomenological threshold values and evolution parameters as the helium fraction in the core [11,12]. This comparison is currently impossible, since modeling has first to accurately reproduce the observed evolutionary tracks. Independent of this forthcoming analysis, we note that the number of stars with ambiguous identification represents less than $1 \%$ of the stars in the clump. Among those, we have identified two high-mass stars that should be in a blue-loop stage.

\section{References}

1. Beck, P. G., Montalban, J., Kallinger, T., et al., Nature 481, 55 (2012)

2. Bedding, T. R., Mosser, B., Huber, D., et al., Nature 471, 608 (2011)

3. Benomar, O., Bedding, T. R., Mosser, B., et al., ApJ 767, 158 (2013)

4. Benomar, O., Belkacem, K., Bedding, T. R., et al., ApJL 781, L29 (2014)

5. Bildsten, L., Paxton, B., Moore, K., \& Macias, P. J., ApJL 744, L6 (2012)

6. Chaplin, W. J., Kjeldsen, H., Christensen-Dalsgaard, J., et al., Science 332, 213 (2011)

7. Deheuvels, S., Doğan, G., Goupil, M. J., et al., A\&A 564, A27 (2014)

8. Girardi, L., MNRAS 308, 818 (1999)

9. Goupil, M. J., Mosser, B., Marques, J. P., et al., A\&A 549, A75 (2013)

10. Huber, D., Silva Aguirre, V., Matthews, J. M., et al., ApJS 211, 2 (2014)

11. Lagarde, N., Decressin, T., Charbonnel, C., et al., A\&A 543, A108 (2012)

12. Montalbán, J., Miglio, A., Noels, A., et al., ApJ 766, 118 (2013)

13. Mosser, B., Barban, C., Montalbán, J., et al., A\&A 532, A86 (2011)

14. Mosser, B., Belkacem, K., Goupil, M., et al., A\&A 525, L9 (2011)

15. Mosser, B., Elsworth, Y., Hekker, S., et al., A\&A 537, A30 (2012)

16. Mosser, B., Goupil, M. J., Belkacem, K., et al., A\&A 540, A143 (2012)

17. Mosser, B., Goupil, M. J., Belkacem, K., et al., A\&A 548, A10 (2012)

18. Mosser, B., Michel, E., Belkacem, K., et al., A\&A 550, A126 (2013)

19. Mosser, B., Benomar, O., Belkacem, K., et al., A\&A 572, L5 (2014)

20. Noels, A. \& Montalbán, J., in Astronomical Society of the Pacific Conference Series, Vol. 479, ed. Shibahashi \& Lynas-Gray, 435 (2013)

21. Stello, D., Huber, D., Bedding, T. R., et al., ApJL 765, L41 (2013)

22. Unno, W., Osaki, Y., Ando, H., Saio, H., \& Shibahashi, H., Nonradial oscillations of stars (University of Tokyo Press, Tokyo, 1989). 